\title{
Aumento de Polifenoles en el Sustrato de Algarrobillo (Samanea saman (Jacq.) Merr.) mediante Fermentación en Estado Sólido con Rhizopus sp (CMPUJ H041)
}

\author{
José E. Mazo(1)*, Dianis Cantillo(1), Kellys Rodríguez ${ }^{(2)}$, y Jayr A. Yepes(3) \\ (1) Facultad de Ingenierías y Tecnológicas, Dpto. de Ing. Agroindustrial, Univ. Popular del Cesar, \\ Valledupar-Colombia. (e-mail: josemazo@unicesar.edu.co). \\ (2) Facultad de Ciencias básicas, Dpto. de Biología, Univ. De La Guajira, Riohacha-Colombia. \\ (e-mail: krodriguezescobar@gmail.com). \\ (3) Facultad de Ciencias de la Salud, Dpto. de Microbiología, Univ. Popular del Cesar, Valledupar- \\ Colombia. (e-mail: jayryepes@unicesar.edu.co).
}

* autor a quien debe ser dirigida la correspondencia

Recibido Abr. 25, 2016; Aceptado Jun. 7, 2016; Versión final Jul. 6, 2016, Publicado Dic. 2016

\begin{abstract}
Resumen
El objetivo de este trabajo fue aumentar polifenoles en el sustrato del fruto de algarrobillo mediante fermentación en estado sólido (FEZ) con Rhizopus sp. (CMPUJ H041). Las condiciones del sustrato fueron $125^{\circ} \mathrm{C}$ y $150 \mathrm{kPa}$ durante $20 \mathrm{~min}$ y $180 \mu \mathrm{m}$. Posteriormente, se establecieron dos muestras (con y $\sin$ $\left.\left(\mathrm{NH}_{4}\right)_{2} \mathrm{SO}_{4}\right)$ de $150 \mathrm{~g}$, aplicando un diseño estadístico completamente al azar con tres repeticiones. A estas muestras se les adicionó la solución nutriente y el inoculo con concentración de esporas $4 \times 10^{6}$ esporas $/ \mathrm{mL}$, y luego se procedió a la FES. Los polifenoles fueron determinados colorimétricamente usando el método de Folin-Ciocalteu. El contenido de polifenoles se incrementó durante la FES y los resultados fueron estadísticamente significativos $(p<0.05)$ obteniéndose $7.324 \mathrm{mg} \mathrm{EAG/g}$ de peso seco de muestra (sin $\left(\mathrm{NH}_{4}\right)_{2} \mathrm{SO}_{4}$ ) y $11.74 \mathrm{mg}$ EAG/g de peso seco (con $\left(\mathrm{NH}_{4}\right)_{2} \mathrm{SO}_{4}$ ). La FES incrementó los compuestos polifenólicos del sustrato, lo que es importante para posibles usos en fuentes de consumo.
\end{abstract}

\section{Increased Polyphenols in Algarrobillo substrate (Samanea saman (Jacq.) Merr.) through Solid State Fermentation with Rhizopus sp (CMPUJ H041)}

\begin{abstract}
The objective of this study was to increase polyphenols in the fruit of algarrobillo substrate through of the solid state fermentation (SSF) with Rhizopus sp. (CMPUJ H041). Substrate conditions were $125^{\circ} \mathrm{C}$ and 150 $\mathrm{kPa}$ during $20 \mathrm{~min}$. and $180 \mu \mathrm{m}$. Subsequently, two samples of $150 \mathrm{~g}$ were drawn (with and without (NH4) 2SO4) and a completely randomized statistical design with three replications was applied. To these samples a nutrient solution and inoculum with spore concentration $4 \times 10^{6}$ spores $/ \mathrm{mL}$ were added, to then proceed with the SSF. Polyphenols were determined calorimetrically using the Folin-Ciocalteu method. The polyphenol content increased and the results were statistically significant $(p<0.05)$ obtaining $7.324 \mathrm{mg}$ EAG/g dry weight (without $\left(\mathrm{NH}_{4}\right)_{2} \mathrm{SO}_{4}$ ) to $11.74 \mathrm{mg} \mathrm{EAG/g}$ dry weight (with $\left(\mathrm{NH}_{4}\right)_{2} \mathrm{SO}_{4}$ ). SSF polyphenolic compounds enhanced total substrate, which is important for possible uses in consumption sources.
\end{abstract}

Keywords: Samanea saman, polyphenol, Rhizopus sp., solid state fermentation 


\section{INTRODUCCIÓN}

El fruto de algarrobillo (Samanea saman (Jacq.) Merr.), es una de las leguminosas que se desarrollan y se encuentran ampliamente distribuidas en el departamento del Cesar, Colombia (SIB, 2015); este fruto contiene azúcares totales $(18.00-30.00 \%$ ) y proteína $(16.08-30.00 \%)$ en peso seco (Jetana et al., 2010), además, existen estudios del fruto donde se acreditan las potencialidades de las semillas con proteínas en respuestas biológicas específicas en la dieta humana (Ogunwande et al., 2006; Sowjanya et al., 2014).

Debido a este tipo de características que tienen ciertas fuentes de leguminosas, se han intensificado investigaciones en la búsqueda de incrementar compuestos funcionales como los polifenoles, que conlleven al mejoramiento y disminución del riesgo de enfermedades futuras en el ser humano (Martins et al., 2011); además, estos son considerados los antioxidantes naturales, que han tenido una gran atención y aceptación por parte de nutricionistas, científicos y consumidores de alimentos, en gran medida, debido a sus funciones en la salud humana. También actúan en la prevención de enfermedades degenerativas tales como cáncer y enfermedades cardiovasculares emergentes (Martins et al., 2011). Debido a estas capacidades funcionales mencionadas han realizado investigaciones para encontrar ciertos componentes fenólicos bioactivos de frutas, plantas y residuos agroindustriales vegetales (Granda et al., 2005), favorecimiento la prevención de enfermedades degenerativas.

Para este tipo de obtención de productos mencionados anteriormente existen procesos biotecnológicos ventajosos frente a la productividad volumétrica del compuesto de interés, ahorro energético que permiten un desempeño adecuado y económico como es la fermentación en estado sólido -FES (Oviedo, J. et al., 2014), la cual, tiene la su capacidad de proporcionar extractos con alta calidad y activada, sin causar efectos secundarios como toxicidad asociado a los solventes orgánicos o los utilizados en otras técnicas de extracción para compuestos polifenólicos (Martins et al., 2011), además es considerado un proceso alternativo para el aumento y producción de compuestos polifenólicos y antioxidantes (Oliveira et al., 2012); este mecanismo fermentativo es importante por qué permite la interacción de ciertas variables como el crecimiento y metabolismo de los microorganismos, composición y propiedades físicas del sustrato y las ambientales (oxígeno, temperatura, actividad de agua y $\mathrm{pH}$ ). Además de lo anterior tiene otras características como la facilidad del manejo, materiales de bajo costo, poco uso de agua entre otros. De acuerdo a estas cualidades del proceso de FES, su funcionalidad y utilidad dependerá del sustrato o material sólido, soporte fuente de nutriente y el microorganismo (Oviedo, J. et al., 2014); para el caso el aumento de polifenoles totales los hongos filamentosos tienen un gran potencial para producir compuestos bioactivos mediante de la FES, y por lo tanto son los microorganismos más utilizados para este propósito (Chen, 2013; Martins et al., 2011). Para este caso específico uno de los hongos considerado uno de los más prometedores en este proceso FES para la obtención de polifenoles es el Rhizopus $s p$., ya que se ha demostrado que tiene la capacidad de aumentar el contenido de proteína de las materias primas de bajo valor nutricional, y con actividad funcional y catalítica específica. Además este microorganismo posee ciertas enzimas que liberan los compuestos fenólicos de forma soluble (Bhanja, T. et al., 2009).También este género no producen sustancias tóxicas y se desempeñarse bien en este tipo de condiciones de fermentación con ausencia de agua, para producir productos bioactivos (Oliveira et al., 2010).

Algunos avances de FES para el aumento del polifenoles encontrados son con semillas de plantas leguminosas como Almorta (Lathyrus sativus) que contienen sabores agradables y con alto contenido nutricional en estado fresco y cocido, utilizando Rhizopus oligosporus DSM 1964, logrando aumentar las propiedades antioxidantes (contenido de compuestos fenólicos) de estas (Starzyńska-Janiszewaka et al., 2008). Así mismo, Lee, I. H., Hung, Y. H., y Chou, C. C. (2008), evaluaron la actividad antioxidante de frijol negro y frijol negro koji preparado con diversos hongos (Aspergillus awamori, Aspergillus oryzae, Aspergillus sojae, Rhizopus azygosporus and Rhizopus sp); evaluaron la propagación de micelios sobre los frijoles y los cambios en el contenido de fenoles totales y de antocianina durante una FES.

Similarmente, se han desarrollado estudios comparativos del contenido fenólico total y potencial antioxidante entre extractos etanólicos de granos de trigo sin fermentar con los fermentados en estado sólido utilizando hongos filamentosos (Aspergillus oryzae and Aspergillus awamori nakazawa. A. oryzae), donde se logró incrementar la concentración de fenoles y propiedades antioxidante frente a los no fermentados (Bhanja, T. et al., 2009).

A esto se le suma la demanda y aceptación por parte de los consumidores de productos procedentes de procesos y fuentes naturales (Alvis, A. et al., 2012), por lo cual la generación de valor agregado del fruto de algarrobillo permitirá darle un giro económico departamental con orientaciones de usos diferenciales que le abran un espacio cultural y social de aplicabilidad como fuentes alimenticias. Por consiguiente el objetivo de esta investigación fue aumentar polifenoles en el sustrato del fruto de algarrobillo mediante fermentación en estado sólido con Rhizopus sp. (CMPUJ H041). 


\section{MATERIALES Y MÉTODOS}

Se explica el tratamiento inicial de los frutos para formar un sustrato y como se hace la caracterización físico química del sustrato y la determinación del porcentaje de proteína, de extracto etéreo de cenizas y de azúcares reductores totales. Luego se detalla la preparación del inóculo, la fermentación en estado sólido y la determinación de contenido de polifenoles totales

\section{Condiciones del sustrato}

Las muestras de frutos de algarrobillo se les realizó un tratamiento de cocción a $125^{\circ} \mathrm{C}$ a $150 \mathrm{kPa}$ durante 20 min, secado $a 60^{\circ} \mathrm{C}$ durante 12 horas y reducción de tamaño de partícula a180 $\mu \mathrm{m}$ con un tamiz en acero inoxidable (TAM-AC8-50) para obtener un sustrato, para su posterior determinación físico química.

\section{Caracterización físico química del sustrato}

La caracterización se llevó a cabo con un análisis que incluyó proteína bruta (NTC 4657) método Kjeldah con el sistema de destilación Kjeldahl marca BUCHI (Unidad de destilación B-324, de digestión K-424 y Scrubber B-414), cenizas (NTC 5167) con la mufla MFR-2001, humedad (NTC 4888) en la Estufa de convección natural ED 23 marca BINDER, azúcares reductores totales con el método del DNS (Miller, 1959) con el espectrofotómetro UV-Visible (Cole-Parmer ${ }^{\circledR}$ Scanning UV / Visible, Vernon Hill, USA), Extracto etéreo con el Método Soxhlet (SM 5520D) B-811marca BUCHI.

\section{Determinación del porcentaje de proteína}

El contenido de proteína en las muestras del sustrato fue calculado con respecto a la ecuación (1)

$$
\mathrm{P}(\%)=6.25 \times[(0.014) \times \mathrm{N} \times \mathrm{V} \times 100) / \mathrm{m}]
$$

En esta ecuación, $\mathrm{P}$ es el porcentaje de proteína; 6.25 = convertidor de gramos de nitrógeno a gramos de proteína; $\mathrm{N}$ = normalidad del ácido; $\mathrm{V}$ = volumen gastados en la titulación - volumen gastados en el problema; $\mathrm{m}=$ masa de la muestra.

\section{Determinación del porcentaje de extracto etéreo}

El contenido de porcentual de extracto etéreo fue calculado con la ecuación (2)

$$
\mathrm{G}(\%)=[(\mathrm{PG}-\mathrm{PV}) / \mathrm{PM}] \mathrm{x} 100
$$

Aquí, $G$ es el porcentaje de extracto estéreo de la muestra; $P G=$ peso del vaso con grasa; $P V=$ peso del vaso vacío; $\mathrm{PM}=$ peso de la muestra (sustrato) en $\mathrm{g}$.

\section{Determinación del porcentaje de cenizas}

El contenido porcentual de cenizas del sustrato fue calculado con respecto a la ecuación (3)

$$
\mathrm{CT}(\%)=[(\mathrm{MCYC}-\mathrm{MCV}) /(\mathrm{MCYMS}-\mathrm{MCV})] \times 100
$$

Siendo CT el porcentaje de cenizas de la muestra; $M C Y C$ = masa del crisol y la muestra calcinada en g; $\mathrm{MCV}=$ masa del crisol vacío en $\mathrm{g} ; \mathrm{MCYMS}=$ masa del crisol y la muestra seca en $\mathrm{g}$.

\section{Azúcares reductores totales}

La absorbancia fue medida en un espectrofotómetro UV-Visible (Cole-Parmer® Scanning UV / Visible, Vernon Hill, USA) a $540 \mathrm{~nm}$. Se preparó una curva patrón de glucosa con las siguientes concentraciones $0.00,0.04,0.08,0.12,0.16,0.20,0.24,0.28,0.32,0.36,0.40 \mathrm{mg} / \mathrm{mL}$ y se desarrolló la reacción con el DNS.

\section{Preparación del inóculo}

Las cepas de Rhizopus sp., (CMPUJ H041) fue suministrada por la Pontificia Universidad Javeriana. Estas se conservaron en Agar Papa Dextrosa (PDA) a $4^{\circ} \mathrm{C}$ previo a su utilización. Posterior a esto, se reactivaron y prepararon las cepas del hongo en cajas de petri con agar PDA, para la obtención de esporas durante 7 días a $30^{\circ} \mathrm{C}$. Luego, se prepararon $30 \mathrm{~mL}$ de Tween 80 al $2.0 \% \mathrm{v} / \mathrm{v}$ que se adicionaron al cultivo y así se 
extrajo la suspensión de esporas. El conteo de esporas se realizó en cámara de Neubauer y se obtuvo una concentración de 4 × $10^{6}$ esporas/mL del inóculo (Oliveira et al., 2011).

\section{Fermentación en estado sólido.}

Se utilizaron dos tratamientos que consistieron de $150 \mathrm{~g}$ de sustrato del fruto de algarrobillo a los cuales se le adicionó una solución nutritiva $(50 \% \mathrm{p} / \mathrm{v})$ con y sin sulfato de amonio $\left(1 \mathrm{~g} / \mathrm{L} \mathrm{KH}_{2} \mathrm{PO}_{4} ; 0.5 \mathrm{~g} / \mathrm{L} \mathrm{MgSO} 4\right.$ y 8 $\left.\mathrm{g} / \mathrm{L}\left(\mathrm{NH}_{4}\right)_{2} \mathrm{SO}_{4}\right)$ y se homogenizó manteniendo la esterilidad (Oliveira et al., 2011). Las fracciones del sustrato fueron vertidas en un prototipo de fermentador previamente esterilizado, donde se realizó el proceso de fermentación en estado sólido, con las características planteadas y aplicadas por Ito, K., et al. (2011), el cual consiste en un sistema formado por una caja de madera abierto en su parte inferior y superior, que permitió que se le introdujera un molde circular provisto de una malla de acero inoxidable, que se cubrió con membranas de politetrafluoroetileno (PTFE) en las partes descubiertas de la caja, para permitir la permeabilidad del vapor generado por el metabolismo del microorganismo durante la FES y evitar la contaminación. Las esporas del hongo Rhizopus sp., (CMPUJ H041) fueron adicionadas en una concentración de $4 \times 10^{6}$ esporas $/ \mathrm{mL}$ (Dey, T., y Kuhad, R., 2014) y previamente homogenizadas. La incubación se realizó en un espacio con poca luminosidad a $30^{\circ} \mathrm{C}$ durante $96 \mathrm{~h}$. La biomasa fermentada se recolectó en envases de plásticos y fue almacenada a $-18^{\circ} \mathrm{C}$.

Los experimentos se hicieron en base a un diseño experimental completamente al azar (DCA) con tres repeticiones para cada uno de los tratamientos; para el primer tratameinto la variable de entrada fue el sustrato con solución nutritiva sin sulfato y la variable de respuesta polifenoles, y el segundo tratamiento la variable de entrada fue el sustrato con solución nutritiva con sulfato de amonio y la variable de respuesta polifenoles, el análisis estadístico se realizó con el programa Statgraphics® Centurion XVI, Marca Registrada (2009). Statpoint Technologies, Inc., U.S.A.

\section{Determinación de contenido de polifenoles totales}

Se pesaron aproximadamente $100 \mathrm{mg}$ por triplicado de la muestra, luego se realizó una extracción de 30 minutos por sonicación con solución extractora de etanol al 70 \% (v/v), el extracto obtenido se llevó a balón volumétrico $2 \mathrm{~mL}$ y se aforo con solución extractora; los extractos obtenidos se les realizó una dilución (1 a 5) y el análisis del contenido de compuestos fenólicos se realizó por el ensayo de Folin Ciocalteu, donde, los polifenoles fueron determinados colorimétricamente usando el reactivo Folin-Ciocalteau (Jiménez et al., 2015). El reactivo contiene ácido fosfo-tungstico como oxidante, que en un medio alcalino es fácilmente reducido por grupos fenólicos produciendo una coloración azul con una absorbancia máxima a $765 \mathrm{~nm}$, cuantificada espectrofotométricamente con base en una curva de calibración empleando 3,4,5- ácido trihidroxibenzoico (Ácido Gálico) como estándar de referencia de lote BCBK2434V, Fluka Analytica (SIGMAALDRICH).

El contenido de polifenoles totales en las muestras del sustrato sin FES y con FES fue calculado con respecto a la ecuación (4)

$\operatorname{PTG}(\%)=\left[\left(\mathrm{PTF}-\mathrm{PTF}_{0}\right) / \mathrm{PTF}_{0}\right] \times 100$

En la ecn. (4), PTG es el porcentaje de polifenoles totales ganados en base seca del sustrato fermentado; PTF $=$ contenido de polifenoles totales en el sustrato fermentado; PTF $_{0}=$ contenido de polifenoles totales en el sustrato sin fermentar.

\section{RESULTADOS Y DISCUSIÓN}

\section{Caracterización físico química del sustrato}

El sustrato del fruto de algarrobillo mostró alto contenido de proteína como fuente de nitrógeno en base seca y azúcares reductores, componentes de interés fundamentales para procesos fermentativos que influyen de manera directa en el desempeño del microorganismo para la obtención de los compuesto polifenólicos, en la tabla 1 , se establecen estos y otros con sus respectivas comparaciones con otros autores. El porcentaje de proteína del sustrato (17.48\%) estuvo dentro del rango (14.10-18.10\%) conseguido por otros autores como Anantasook y Wanapat (2012) y Semae et al (2013), de igual manera el porcentaje de cenizas (4.06\%) es cercano a valores reportados por Anantasook y Wanapat (2012) con $3.19 \%$ y Hosamani, S. V. et al (2005) con $4.50 \%$. 
Tabla 1. Caracterización físico química del sustrato del fruto de algarrobillo y su respectiva comparación con otros estudios. El \% de sustrato es la media \pm desviación estándar

\begin{tabular}{|l|c|c|l|}
\hline \multicolumn{1}{|c|}{ Componente $(\mathrm{g} / \mathrm{100 \textrm {g } )}$} & $\begin{array}{c}\text { Sustrato }(180 \mu \mathrm{m}) \\
\text { Resultado* }(\%)\end{array}$ & Comparación (\%) & \multicolumn{1}{|c|}{ Referencia } \\
\hline Proteína Estimada (N) & $19.7 \pm 0.002$ & $14.10-20.10$ & $\begin{array}{l}\text { Jetana et al., 2010; } \\
\text { Semae, et al., 2013 }\end{array}$ \\
\hline Azúcares reductores totales & $24.7 \pm 0.008$ & $18.00-30.00$ & Jetana et al., 2010 \\
\hline Extracto etéreo & $1.03 \pm 0.0004$ & $1.69-2.67$ & Semae et al., 2013 \\
\hline Cenizas & $4.06 \pm 0.0094$ & $3.19-4.5$ & Anantasook y Wanapat, 2012 \\
\hline Polifenoles totales & $7.015 \% \pm 0.2915$ & - & - \\
\hline
\end{tabular}

El extracto etéreo (1.10\%) está por debajo de los rangos $(1.40-2.67 \%)$ reportados por Anantasook y Wanapat, (2012) y Semae et al., (2013), los azúcares reductores totales (24,74\%) se encuentran dentro del rango de 10 - 30\% hallado por Hosamani, S. V. et al (2005) y Jetana et al., (2010), este contenido es causado por el tratamiento físico previo (cocción y reducción de tamaño) que fue sometido, aumentando la concentraciones de los azúcares y arrastres de estos por el agua y su facilidad para su determinación, también logró la homogeneidad del sustrato y en especial el desprendimiento de los azúcares que se encuentran en la vaina del fruto.

\section{Fermentación en estado sólido}

El proceso de FES con sulfato de amonio permitió el aumento de los polifenoles totales en $4.71 \mathrm{mg} \mathrm{EAG/g}$ (equivalentes de ácido gálico) en peso seco, correspondiente a un $67.30 \%$ con incremento en el sustrato del fruto de algarrobillo (tabla 2), frente a los tratamientos sin sulfato de amonio y el testigo; estos valores están por encima de los reportados por Schmidt y Furlong (2012), donde obtuvieron con salvado de arroz polifenoles totales de $4 \mathrm{mg}$ ácido ferúlico/ peso seco y por debajo frente a los reportados por Bhanja, T., y Kuhad, R. C., (2014) donde obtuvieron con trigo polifenoles totales de $6.78 \mathrm{mg} \mathrm{EAG/g}$ en peso seco.

Tabla 2. Polifenoles totales posterior a la fermentación con y sin adición de sulfato de amonio

\begin{tabular}{|c|c|c|}
\hline Tratamiento & $P(\mathrm{mg}$ EAG/g de peso seco) & PAP (\%) \\
\hline FSS & $0.31 \pm 0.20$ & $4.4 \pm 0.20$ \\
\hline FCS & $4.72 \pm 0.47$ & $67.30 \pm 0.47$ \\
\hline
\end{tabular}

En la Tabla 2, $\mathrm{P}$ son los polifenoles en peso seco (expresados en miligramos de equivalente de ácido gálico EAG); PAP es el porcentaje de aumento de polifenoles totales; FSS son los Tratamientos fermentados sin $\left(\mathrm{NH}_{4}\right)_{2} \mathrm{SO}_{4}$; FCS son los Tratamientos fermentados con $\left(\mathrm{NH}_{4}\right)_{2} \mathrm{SO}_{4}$. De acuerdo al análisis estadístico de pruebas de rangos de Tukey, arrojó que existen diferencias significativas $(p \leq 0.05)$ entre FCS con respecto al testigo y FSS, mientras que el testigo y FSS no.

El suministro de sulfato de amonio al $8 \mathrm{~g} / \mathrm{L}$ en la solución nutriente generó una gran variación frente al tratamiento sin sulfato de amonio, favoreciendo con el aumento de $67.30 \%$ de polifenoles (tabla 2).

Las características del sustrato, en particular su alto contenido de azúcares reductores, sustentó el crecimiento del hongo y permitió el aumento de polifenoles en el tratamiento con sulfato de amonio, posiblemente debido a que estos compuestos se encuentran en forma conjugada con azúcares, ácidos grasos o aminoácidos y la acción del microorganismos permitió su liberación . Las enzimas carbohidrasas producidas por el hongo como celulasas, $\beta$-glucosidasa, xilanasa, pectinasas, $\beta$-xilosidasa, $\beta$-galactosidasa y $\alpha$-amilasas pueden liberar los compuestos fenólicos unidos hasta una forma soluble. También, esto puede ser debido al hecho de que las enzimas hidrolíticas producidos por hongos, (Xilanasas, $\alpha$ amilasas, y $\beta$ glucosidasas) catalizan la liberación de agliconas del sustrato de ese modo resulta en un aumento de su contenido fenólico. En este estudio se demuestra que las condiciones del sustrato permitió el incrementó el contenido de polifenoles totales.

Por otra parte, la FES sin sulfato de amonio el aumento de polifenoles totales fue menor $(4,40 \%)$ para un $0.30 \mathrm{mg} \mathrm{EAG} / \mathrm{g}$ en peso seco. Por consiguiente, el suministro de fuente exógena de nitrógeno favorece al incremento de polifenoles totales (figura 1). 


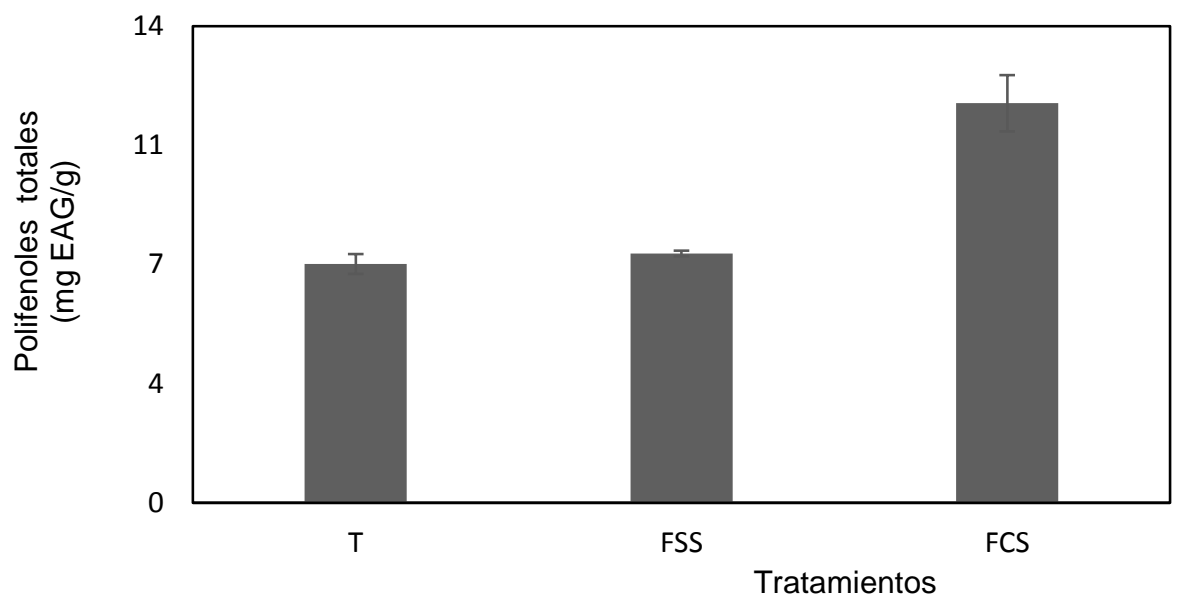

Fig. 1: Resultados comparativos de los polifenoles totales de los tratamientos, donde: $\mathrm{T}=$ Testigo sin fermentar; FSS = Fermentación $\sin \left(\mathrm{NH}_{4}\right)_{2} \mathrm{SO}_{4} ; \mathrm{FCS}=$ fermentado con $\left(\mathrm{NH}_{4}\right)_{2} \mathrm{SO}_{4}$.

\section{Efecto del sistema de fermentación FES}

El sistema de fermentación adaptado de Ito et al (2011), fue determinante para la interrelación entre las propiedades del sustrato, el metabolismo del microorganismo, los factores y el medio donde se realizó la fermentación, permitieron el incremento de los polifenoles durante el periodo de fermentación. La uniformidad del cultivo en un prototipo sin aireación cuya aplicación solo ha sido para la obtención de enzimas y su optimización, en este caso permitió que el microorganismo se desempeñara adecuadamente con respecto al aumento de compuestos polifenólicos.

\section{CONCLUSIONES}

Esta investigación es la base inicial del proceso de fermentación en estado sólido (FES) para el aumento de compuestos de polifenoles totales en el fruto de algarrobillo como sustrato, considerándose adecuado para este tipo de sustrato con una especificación dirigida al aumento de compuestos polifenólicos.

El estudio indica que el proceso de fermentación en estado sólido en un sistema cerrado y sin aireación puede ser utilizado para mejorar las características nutricionales del fruto de algarrobillo (sustrato).

Este proceso demostró que el sustrato de algarrobillo fermentado con Rhizopus sp., (CMPUJ H041) con fuentes exógenas de nitrógeno favoreció al aumento de polifenoles totales.

La FES incrementó los compuestos polifenólicos del sustrato, por lo tanto, es importante para potenciales aplicaciones en la agroindustria de balanceados para animales, farmacéutica, o de alimentos.

\section{AGRADECIMIENTOS}

Los autores agradecen a la Universidad Popular del Cesar a través de los grupos de investigación Energías Alternativa y Biomasa GEAB, Microbiología Ambiental y Agrícola MAGYA, y el GISB de la Universidad de Antioquia.

\section{REFERENCIAS}

Anantasook, N., y Wanapat, M. Influence of rain tree pod meal supplementation on rice straw based diets using in vitro gas fermentation technique, doi: http://dx.doi.org/10.5713/ajas.2011.11131, Asian-Australasian Journal of Animal Sciences, 25(3), 325-334 (2012)

Alvis, A., Martínez, W. y Arrazola, G. Obtención de Extractos Hidro-Alcohólicos de Limoncillo (Cymbopogon citratus) como Antioxidante, doi: 10.4067/S0718-07642012000200002, Información Tecnológica, 23(2), 3-10 (2012) 
Bhanja, T. y Kuhad, R. C. Enhanced production and extraction of phenolic compounds from wheat by solidstate fermentation with Rhizopus oryzae RCK2012, doi:10.1016/j.btre.2014.09.006, Biotechnology Reports, 4, 120-127 (2014)

Bhanja, T. Kumari, A. y Banerjee, R. Bioresource Technology Enrichment of phenolics and free radical scavenging property of wheat koji prepared with two filamentous fungi, doi:10.1016/j.biortech.2008.12.055, Bioresource Technology, 100(11), 2861-2866 (2009)

Chen, H. Modern Solid State Fermentation Theory and Practice, 32-33. Springer Dordrecht Heidelberg New York London (2013)

Dey, T., y Kuhad, R. Enhanced production and extraction of phenolic compounds from wheat by solid-state fermentation with Rhizopus oryzae RCK2012, doi:10.1016/j.btre.2014.09.006, Biotechnology Reports, 4, 120 $-127(2014)$

Granda, D. Mejía, A. Jiménez, G. Utilización de residuos de plátano para la producción de metabolitos secundarios por fermentación en estado sólido con el hongo Lentinus crinitus, http://www.scielo.org.co/pdf/vitae/v12n2/v12n2a02.pdf; ISNN: 0121-4004, Vitae, revista de la facultad de química farmacéutica: 12(2), 13-20 (2005)

Hosamani, S. V., Gowda, N. K. y Kololgi, S. D. Evaluation of Chemical , Nutritive and Feeding Value of Rain Tree Pods, http://14.139.155.167/test5/index.php/kjas/article/viewFile/3031/3260, Karnataka J. Agric. Sci: 18(1), 110-113 (2005)

Ito, K., Kawase, T., Sammoto, H., Gomi, K., Kariyama, M., y Miyake, T. Uniform culture in solid-state fermentation with fungi and its efficient enzyme production, doi:10.1016/j.jbiosc.2010.11.008, Journal of Bioscience and Bioengineering. 111(3), 300-305 (2011)

Jetana, T., Vongpipatana, C., Thongruay, S., Usawang, S., y Sophon, S. Apparent digestibility, nitrogen balance, ruminai microbial nitrogen production and blood metabolites in Thai Brahman cattle fed a basal diet of rice straw and supplemented with some tropical protein-rich trees, doi:10.5713/ajas.2010.90316, AsianAustralasian Journal of Animal Sciences: 23(4), 465-474 (2010)

Jiménez, N., Carrillo-Hormaza, L., Pujol, A., Álzate, F., Osorio, E., y Lara-Guzman, O. Antioxidant capacity and phenolic content of commonly used anti-inflammatory medicinal plants in Colombia, doi:10.1016/j.indcrop.2015.03.050, Industrial Crops and Products: 70, 272-279 (2015)

Lee, I. H., Hung, Y. H., y Chou, C. C. Solid-state fermentation with fungi to enhance the antioxidative activity, total phenolic and anthocyanin contents of black bean, doi:10.1016/j.ijfoodmicro.2007.09.008, International Journal of Food Microbiology, 121(2), 150-156 (2008)

Martins, S., Mussatto, S. I., Martínez-Avila, G., Montañez-Saenz, J., Aguilar, C. N., y Teixeira, J. A. Bioactive phenolic compounds: production and extraction by solid-state fermentation, $A$ review, doi:10.1016/j.biotechadv.2011.01.008, Biotechnology Advances, 29(3), 365-73 (2011)

Manach, C., Scalbert, A., Morand, C., Rémésy, C., y Jiménez, L. Polyphenols: Food sources and bioavailability, $\quad$ http://ajcn.nutrition.org/content/79/5/727.full.pdf+html?sid=d821 ac55-c914-489f-904dbdc14e7130b1, ISSN: 0002-9165, American Journal of Clinical Nutrition, 79(5), 727-747 (2004)

NTC, 3594, Norma Técnica Colombiana. Productos de molinería. Harina precocida de maíz para consumo humano, 1-10, Bogotá, Colombia (2014)

NTC, 4657, Norma Técnica Colombiana. Alimento para animales. Determinación del contenido de nitrógeno y cálculo del contenido de proteína cruda, método kjeldahl, 1-5, Bogotá, Colombia (1999)

NTC 4888. (2000). Norma Técnica Colombiana. Alimentos para animales. Determinación del contenido de humedad y materia volátil, 1-7, Bogotá, Colombia (2000)

Ogunwande, I. A, Walker, T. M., Setzer, W. N., y Essien, E. Volatile constituents from Samanae saman (Jacq.) Merr., Fabaceae, doi: 10.5897/AJB2006.000-5081, African J.Biotechnol; 5(20), 1890-1893 (2006)

Oliveira, M. D. S., Feddern, V., Kupski, L., Cipolatti, E. P., Badiale-Furlong, E., y De Souza-Soares, L. A. Changes in lipid, fatty acids and phospholipids composition of whole rice bran after solid-state fungal fermentation, doi:10.1016/j.biortech.2011.06.025, Bioresource Technology; 102(17), 8335-8338 (2011) 
Oliveira, S., Cipolatti, E. P., Furlong, E. B., y Soares, L. D. S. Phenolic compounds and antioxidant activity in fermented rice (Oryza sativa ) bran, http://www.scielo.br/pdf/cta/v32n3/aop_cta_5500.pdf, ISSN: 1678-457X, Ciència E Tecnologia de Alimentos; 32(3), 531-537 (2012)

Oviedo, J., Casas, A., Valencia, J., y José E. Zapata. Análisis de la Medición de la Biomasa en Fermentación en Estado Sólido empleando el Modelo Logístico y Redes Neuronales, doi:10.4067/S071807642014000400016, Información Tecnológica, 25(4), 141-152 (2014)

Schmidt, C. G., y Furlong, E. B. Effect of particle size and ammonium sulfate concentration on rice bran fermentation with the fungus Rhizopus oryzae, doi:10.1016/j.biortech.2012.07.081, Bioresource Technology; 123, 36-41 (2012)

Semae, S., Kongmun, P., y Vajrabukka, C. Effects of Different Levels of Rain Tree ( Samanea saman ) Pods in Meal Concentrate on In Vitro Fermentation by a Gas Production Technique, http://agkb.lib.ku.ac.th/BKN_AGRI/search_detail/result/307618, 711, 704-711 (2013)

SIB, Sistema de Información Sobre Biodiversidad de Colombia. Búsqueda geográfica: Colombia. (En línea: http://data.sibcolombia.net/departamentos/, acceso 12 de Octubre 2015)

Sowjanya, J., y otros seis autores. An overview on the biological perspectives of samanea saman (jacq.)merr (rain tree), www.ijppsjournal.com/Vol6Issue5/9294.pdf, ISSN: 0975-1491, International Journal of Pharmacy and Pharmaceutical Sciences; 6(5), 6-8 (2014)

Starzyńska-Janiszewska, A., Stodolak, B., y Jamróz, M. Antioxidant properties of extracts from fermented and cooked seeds of Polish cultivars of Lathyrus sativus, doi:10.1016/j.foodchem.2007.12.028, Food Chemistry; 109(2), 285-292 (2008) 